\title{
An Enhanced Depth-integrated Model for Flows over a Negative Step with Hydraulic Jump
}

\author{
Tatsuhiko Uchida ${ }^{1}$ \\ ${ }^{1}$ Department of Civil and Environmental Engineering, Graduate School of Engineering, Hiroshima \\ University, Japan
}

\begin{abstract}
Depth-integrated models play an important role of predicting flows for several practical problems. There are many contributions to develop an enhanced depth-integrated model to have the ability of evaluating vertical flow structures. However, it is still challenging for the depth integrated models to calculate flows over a negative step accompanying a hydraulic jump, because the separation zone behind the step and the pattern of the hydraulic jump depend on the several hydraulic conditions such as Froude number, step height and downstream water depth. In this study, assuming gravel bed rivers in which the bed is covered with relatively large roughness, an enhanced depth integrated model is developed to calculate various hydraulic jump patterns generated downstream from the negative step. The present method is based on General Bottom Velocity Computation method employing Dynamic Wall Law with 4th degree polynomial velocity distribution (GBVC4-DWL). The model with the virtual bed slope behind the negative step and the critical slope of wave breaking is validated through the comparisons with the experimental results on flows over a negative step for submerged jet, weak jump and undular jump conditions. Then the essential terms of equations which compose the present method to calculate the flow downstream of the negative step are investigated.
\end{abstract}

\section{Introduction}

The negative steps can be seen in rivers around several structures such as weirs, groundsills, bed protection works and revetment works. Because the river bed downstream from the negative step is prone to be scoured and eroded, it is important for designing river structures and those maintenances to clarify flows over negative steps.

Depth-integrated models have played an important role of predicting flows for several practical problems. Because non-hydrostatic three dimensional models are too expensive to be applied to practical river problems with large scale phenomenon, there are many contributions to develop an enhanced depth-integrated models to have the ability of evaluating vertical flow structures to evaluate velocity distribution and sediment transport for curved and meandering channels, including secondary flow models and quasi-three dimensional models [1-7]. Recently, the authors developed the General Bottom Velocity computation (GBVC) method [8] which is a non-hydrostatic depth integrated model to calculate three dimensional flow and sediment transport induced by the three dimensional 
vortex motions around structures and those in compound meandering channels within the framework of the two-dimensional model. The GBVC4-DWL method [9] is a sophisticated model without assuming equilibrium bottom boundary conditions but with employing Dynamic Wall Law (DWL) to calculate complex flow patterns near the bed induced by the presence of submerged obstacles. However, it is still challenging for the depth integrated models to calculate flows over negative steps accompanying hydraulic jumps, because the separation zone behind the step and the pattern of the hydraulic jumps depend on the several hydraulic conditions such as Froude number, step height and downstream water depth $[10,11]$.

In this study, assuming gravel bed rivers in which the bed is covered with relatively large roughness to the water depth, an enhanced depth integrated model is developed to calculate various hydraulic jump patterns generated downstream from the negative step. The present method is based on GBVC4-DWL method. The method with the virtual bed slope behind the negative step and the critical slope of wave breaking is validated through the comparisons with the experimental results. Then the effects of equations and terms which compose the present method on the flow structures downstream of the negative step are investigated by using several simplified calculation models from GBVC4-DWL including GBVC3 (an equilibrium wall law model), SBVC3 (a hydrostatic model) and 1DC (a pure onedimensional model without considering variations in vertical velocity and pressure distributions).

\section{Calculation method}

The present calculation method is developed based on the General Bottom Velocity Computation method, which is a depth integrated model with the ability to calculate the vertical distributions of horizontal velocity and nohydrostatic pressure [8], employing 4th degree polynomial velocity distribution and Dynamic Wall Law (GBVC4- DWL method) [9]. As shown in Fig. 1, the calculation domain is divided into a main calculation domain for calculating a vertical flow structure and a thin wall law region near the bed. The wall law region consists of a vortex layer and a roughness layer. In this study, the boundary between the main calculation region and the vortex layer is defined as the bottom height $z_{b}$, and the boundary between the vortex layer and the roughness layer is defined as the roughness surface height $z_{t}$. When the riverbed material is made of a permeable material such as gravel, there is an infiltration subsurface layer under the roughness layer [12]. In the experimental condition targeted in this study, the lower surface of the roughness layer is impermeable (see Fig. 3), and there is no flow, so the permeable sublayer is not considered. The equations and unknown variables solved in each region of the GBVC4-DWL method are shown in Table 1.

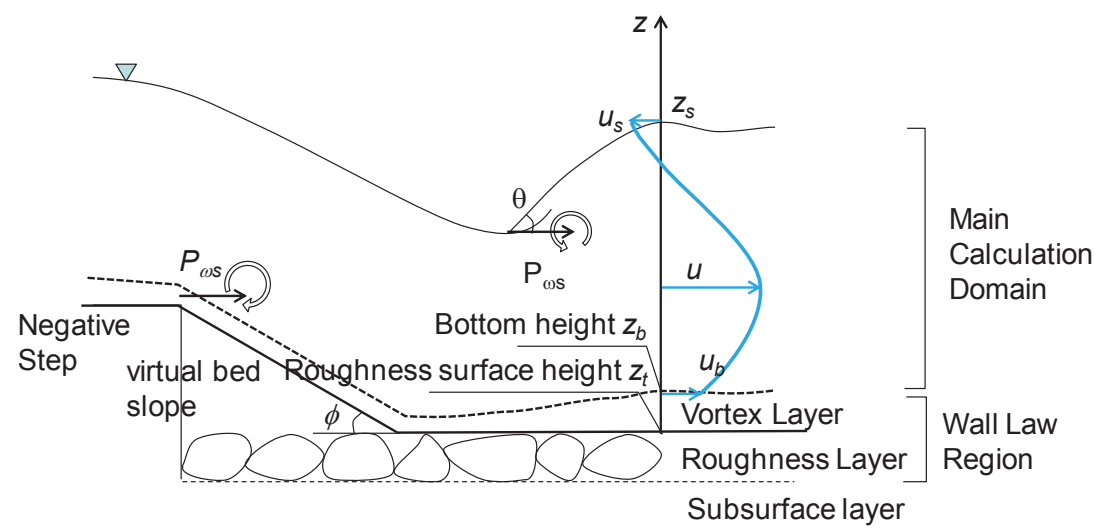

Fig. 1. Calculation domains of GBVC4-DWL method for flow over negative step. 
Table 1. Equations and unknown variables.

\begin{tabular}{|l|l|l|l|l|}
\hline $\begin{array}{c}\text { Calculation } \\
\text { Domain }\end{array}$ & \multicolumn{2}{|c|}{$\begin{array}{c}\text { Calculation } \\
\text { Methods }\end{array}$} & \multicolumn{1}{|c|}{ Enknown variables } & \\
\hline & & & &
\end{tabular}

DI: depth integrated, TKE: turbulence kinetic energy, VL: vortex layer, RL: roughness layer

The role of each equation and term composing GBVC4-DWL on calculations for flows over negative step with a hydraulic jump is investigated though the comparisons with the simplified calculation methods indicated in the Table 1. Those include the one-dimensional calculation (1DC) method based on shallow water flow equations, the Simplified Bottom Velocity Computation (SBVC3) method [7], and the General Bottom Velocity Computation (GBVC3) method [8,9]. The numbers represent the order of the polynomial of the vertical distribution of horizontal velocity for the main calculation domain. The differences between the GBVC4-DWL method and each simplified calculation method are summarized below.

In the GBVC 3 method, the fluxes across the bottom height $z_{b}$ and the roughness surface height $z_{t}$ are assumed to be zero, and the equilibrium conditions are assumed for the flow in the wall law layers. The shear stresses and vortex exchange due to flow turbulence at the bottom height are evaluated by assuming a logarithmic velocity distribution within the thin wall law layer for the boundary condition of the main calculation domain. In the SBVC3 method, which simplifies the GBVC3 method with the assumption of the shallow water flow, the spatial variation term of the vertical flow velocity of the definition for the horizontal vorticity is set to be zero and the hydrostatic pressure distribution is assumed for the momentum equations. In comparison with the SBVC3 method, the 1DC method includes no equations other than the depth integrated equations of continuity, horizontal momentum and TKE equations. In other words, 1DC method calculate equations, assuming the uniform vertical velocity distribution proposed by Engelund [13] instead of calculating vertical velocity distributions. Since the non-hydrostatic pressure component of the pressure represents the non-equilibrium of the vertical flow velocity, the non-equilibrium effects of various quantities on a target flow is possible to be investigated by comparing various calculation methods in Table 1. The detail of the calculation methods and equations in the Table 1 can be found in our previous papers [7-9]. 


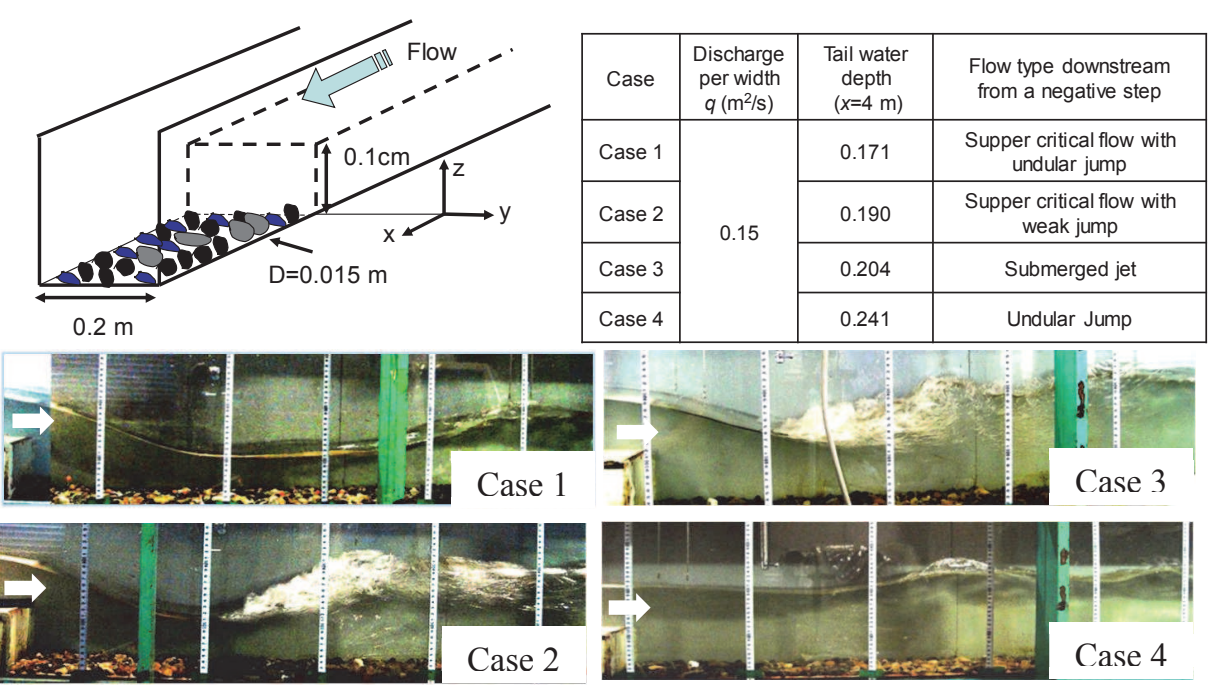

Fig. 2. Experimental setup and cases with hydraulic conditions.

\section{Experimental and Calculation setup}

For the experiment, a straight channel with a length of $6.0 \mathrm{~m}$, a width of $0.20 \mathrm{~m}$ and a slope of $1 / 1000$ was used. A rectangular block with a length of $0.9 \mathrm{~m}$, a width of $0.2 \mathrm{~m}$, and a height of $0.1 \mathrm{~m}$ is installed as a negative step, and the gravel particles with a diameter of $D=15 \mathrm{~mm}$ were fixed on the bed surface as in indicated in Fig.2. In this paper, the $x, z$ axises are taken in the downward and the vertical upward direction, respectively. The origin is set at the bed height of the negative step section. The table in Fig. 2 shows the experimental conditions and flow types investigated downstream of the negative step in the experiment. With the different tail water depth, several hydraulic jumps were induced as indicated in the pictures in Fig.2.

In the numerical calculations, the discharge and the water depth are given at the upstreamend and the downstream-end, respectively, and a rectangular block is installed as in the experiment. The equivalent roughness on the block was set to $k_{s}=0.01 \mathrm{~mm}$. Although the GBVC method can analyze the flow field of the rapidly varied flow over structure with separation zone [9], the calculation of interactions between the water surface profile and separation zone behind a negative step is still challenging issue for the GBVC method, because the depth averaged non-hydrostatic pressure is evaluated by using bottom pressure $p_{b}$. In this research, as shown in Fig. 1, a virtual bed slope behind the negative step is assumed as a separation zone. The slope gradient $\phi$ was adjusted so as to reproduce experimental results. Consequently, $\tan \phi=0.7$ for Case 1 and $2, \tan \phi=0.45$ for Case 3 and $\tan \phi=0.12$ for Case 4 were obtained. For bed roughness downstream the step, $z_{t}=5 \mathrm{~mm}, k_{s}=40 \mathrm{~mm}$ were used. To calculate different types of hydraulic jump with depth integrated model, the critical water surface gradient $\tan \theta_{c}=0.6$ is introduced as the wave breaking condition.

\section{Calculation results and discussions}

Fig. 3 compares the calculation results of water surface profiles by GBVC4-DWL for Case 1-6 with those measured by the experiment. Fig. 4 shows the computational velocity fields colored with the non-hydrostatic pressure distribution. Here, the pressure distribution in Fig. 4 is assumed as a linear distribution in the vertical direction connecting the bottom pressure and zero free surface pressure. For Case 1, a supper critical flow accompanying undular jump 
is formed downstream from the negative step. The calculated water surface profile for Case 1 is in good agreement with that of the experiment. As shown in Fig. 4, the low pressure zone is formed behind the step, and the pressure is high at the water attacking point on the bed accompanying the downstream supper critical flow. At the downstream-end of the supper critical flow zone, the flow transits smoothly to downstream water depth, forming an undular jump. For the undular jump, low and high bottom pressure zone are generated at the crest and trough of waves, respectively. In Case 2, the jump section moves to upstream direction with increasing tail water depth from Case 1. The jump type changes to the weak jump because Fr number of flow to transit is increased by moving upstream. Unlike the smooth water surface profile of the undular jump for Case 1, the water surface of supper critical flow is discontinuously connected to the tail water depth with convex shape hydraulic jump. The above characteristics investigated in the experiment is captured well by the calculation result. For Case 3, the jump section reaches to the separation zone behind the step and the length of the jump is increased. Although the virtual bed slope needs to be decreased to $\tan \phi=0.45$ for the calculation for Case 3, the water surface profile of submerged jet is well reproduced. In the Fig. 4, the reverse flow is formed near the free surface in the submerged jet for Case 3. A undular jump is also investigated for Case 4 just downstream from the step. From Fig. 4 , it is validated that the present method can calculate the undular jump for Case 4 in which the wavy water surface profile is formed with a large separation zone behind the step as shown in Fig. 2. It is also noticed that the water level upstream from the section of the step is marginally increased for Case 4 compared to the other Cases. Because the negative pressure behind the step is recovered due to the undular jump induced just downstream from the step for Case 4. It is validated that the present calculation method with the mild virtual slope with $\tan \phi=0.12$ behind the step show good agreement with the experimental result and explain the flow characteristics investigated in Fig.2 for the undular jump condition of Case 4. As indicated above, GBVC4-DWL method can reproduce flow structures in the hydraulic jumps formed downstream of a negative step within a frame work of one-dimensional calculation method by installing the appropriate virtual slope.
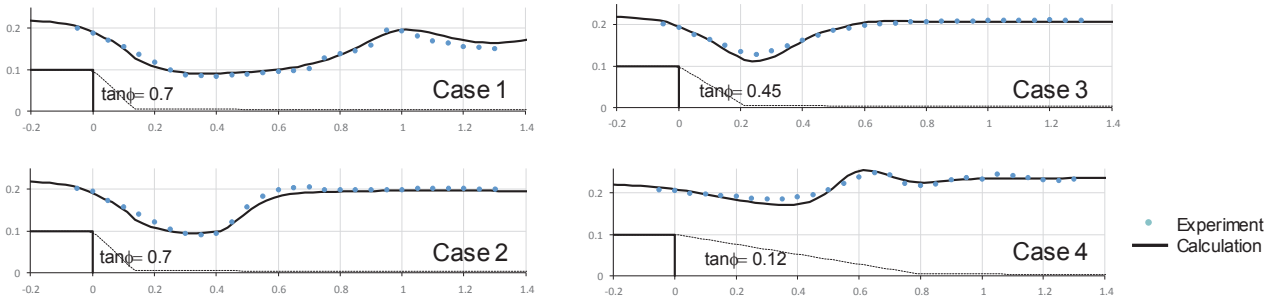

Fig. 3 Comparisons of water surface profiles between the experiments and present calculation results
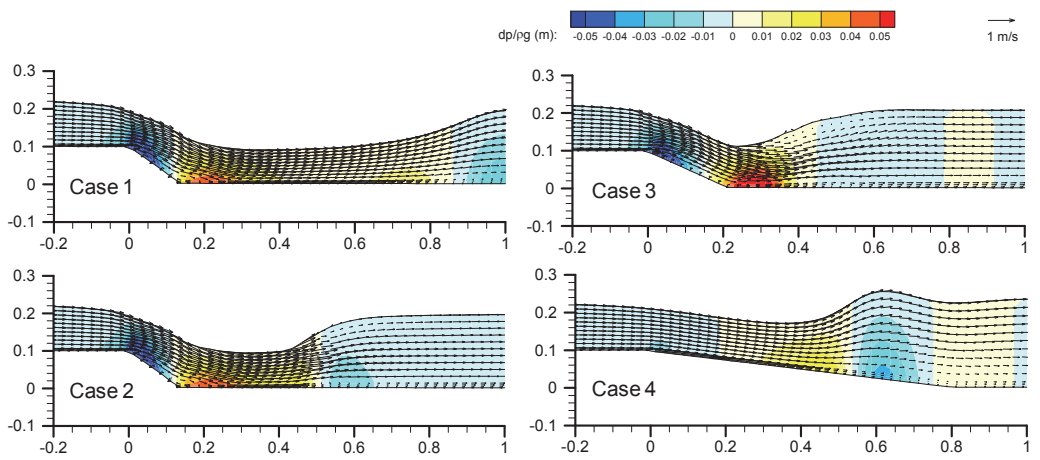

Fig.4 Velocity distributions downstream of a negative step calculated by GBVC4-DWL 
In order to clarify the essential terms and mechanisms to induce different types of hydraulic jumps downstream of the step, Fig.5 shows comparisons of calculated water surface profiles with various calculation methods in Table 1 including GBVC4-DWL, GBVC3, SBVC3 and 1DC conducted under the same computational conditions. The water depth profiles upstream from the step section calculated with 1DC and SBVC3 are higher than those with GBVC3 and GBVC4-DWL, while the lower water levels than GBVC3 and GBVC4-DWL on the separation zone behind the step are calculated with 1DC and SBVC3. The 1DC and SBVC3 methods assume hydrostatic pressure distributions [7], although GBVC3 and GBVC4-DWL calculate non-hydrostatic pressure distributions without the shallow water assumption $[8,9]$. It is clarified that the non-hydrostatic pressure distribution needs to be taken into account to calculate rapidly varied flow over the step. Because there is little difference between 1DC and SBVC3, the velocity distribution due to vortex is not always necessary to calculate rapidly varied flows. The difference in water level upstream from the step between hydrostatic models (1DC and SBVC3) and non-hydrostatic models (GBVC3 and GBVC4-DWL) is decreased with increasing the tail water depth.
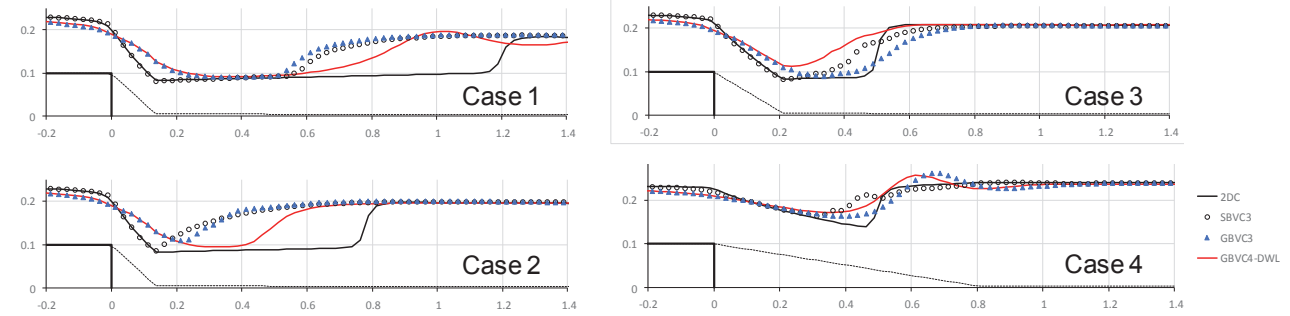

Fig.5 Comparisons of water surface profiles computed with various calculation methods in Table 1

Looking at the water surface profile for Case 1 and 2, the hydraulic jump sections calculated with 1DC is far downstream from those with the other methods. Because 1DC method cannot evaluate the unestablished velocity distribution for the flow downstream from the step, shear stress acting on the bed of 1DC is lower than the other methods and then the supper critical flow can sustain for the longer distance. In addition, 1DC assumes that the energy loss due to hydraulic jump is occurred for very short distance. There are little differences in shapes of hydraulic jumps calculated with 1DC for Case 1 to 4 . Although the calculated water surface profile upstream from the hydraulic jump with SBVC3 method is similar to that with the 1DC method, the section of the hydraulic jump moves upstream. Because the SBVC3 method can evaluate velocity distribution due to vortex produced on the bottom, the bottom velocity downstream from the step for SBVC3 method is higher than that for 1DC method. In addition, the vortex supply and velocity distribution in the jump zone are taken into account in the SBVC3 method, the convex hydraulic jump of weak jump for Case 2 and submerged jet for Case 3 are able to be captured with SBVC3 method. However, the undular jump for Case 1 and 4 cannot be reproduced by SBVC3 method. Considering the fact that the undular jump for Case 4 is reproduced with GBVC3 method, it is concluded that the undular jump is not subjected to non-equilibrium velocity distribution due to vortex production, but induced by non-hydrostatic pressure distribution due to non-equilibrium vertical velocity. Comparing calculated water surfaces between GBVC3 and GBVC4-DWL, there is little difference in water surface profiles upstream from the hydraulic jump section. However, the location of the jump is moved downstream by introducing the dynamic wall law which takes into account non-equilibrium flows in the wall law layer near the bed. As a result, the GBVC3 fails to reproduce the undular jump for Case 1 which is explained with GBVC4-DWL method. It is considered that in the experiment, a spatial variation in the pressure near the bottom just upstream from the jump induces a non-equilibrium flow in the 
gravel layer. Although further investigation is necessary to validate the DWL for flow in the gravel layer, it can be said that DWL has an important role to calculate flow with hydraulic jumps on rough bed in gravel bed rivers. On the other hand, the larger the tail water depth, the smaller the difference of water surface profile between the GBVC3 method and the GBVC4-DWL method. For this reason, the role of non-equilibrium of the flow near the bottom decreases with increasing water depth.

\section{Conclusions}

An enhanced depth integrated model based on GBVC4-DWL was developed to calculate flow structures downstream from a negative step and a hydraulic jump that varies with the change in tail water depth. It was clarified through the comparisons between the experimental and calculation results that the model with the virtual bed slope behind the negative step and the critical slope of wave breaking is able to calculate several types of hydraulic jump such as weak jump, undular jump and submerged jet. The comparisons of GBVC4-DWL method with several simplified calculation models, including GBVC3 (equilibrium wall law model), SBVC3 (hydrostatic model) and 1DC (pure one dimensional model without considering vertical velocity and pressure distributions), revealed the role of equations and terms on calculations for the flows and hydraulic jump downstream of the negative step as follows. The problems of 1DC method include the disabilities to calculate rapidly varied flow over a step and underestimate the resistance for supper critical flow zone downstream of the step. For the calculation of rapidly varied flows, it is indispensable to consider non-hydrostatic pressure component, while the variation in velocity distribution due to the vortex is not dominant. Although weak jump and submerged jet accompanying wave breaking at the upstream of the jump can be calculated by the hydrostatic model with the ability to calculate vertical velocity distribution, the undular jump needs to be calculated with non-hydrostatic model. For the calculation for hydraulic jump on rough bed, because large pressure gradient upstream of the jump induces non-equilibrium flow for the gravel layer, the dynamic wall law (DWL) model is required.

\section{References}

1. Ishikawa, T., Suzuki, K., Tanaka, M. Efficient numerical analysis of an open channel flow with secondary circulations, Proc. JSCE 375/II-6, 181-189 (1986)

2. Fukuoka, S., Watanabe, A., Nishimura, T. On the groin arrangement in meandering rivers. Ann. J. Hydraul. Eng. JSCE 443/II-18, 27-36, (1992)

3. Jin, Y.-C., Steffler, P.M. Predicting flow in curved open channels by depth-averaged method, J. Hydraul. Eng. ASCE 119(1), 109-124 (1993)

4. Yeh, K.-C., Kennedy, J.F. Moment model of nonuniform channel-bend flow. I: fixed beds. J. Hydraul. Eng. ASCE 119(7):776-95, (1993)

5. Finnie, F., Donnell, B., Letter, J., Bernard, S.B. Secondary flow correction for depth averaged flow calculations. J. Eng. Mech. 125, 848-63 (1999)

6. Blanckaert, K., de Vriend, H.J. Nonlinear modeling of mean flow redistribution in curved open channels. Water Resour. Res. 39(12), 1375 (2003)

7. Uchida, T. and Fukuoka, S. Numerical Simulation of Bed Variation in a Channel with a Series of Submerged Groins, Proceedings of the 34th IAHR World Congress, Brisbane, Australia, 4292-4299 (2011) 
8. Uchida, T. and Fukuoka, S. Numerical calculation for bed variation in compoundmeandering channel using depth integrated model without assumption of shallow water flow, Adv. Water Resour. 72, 45-56 (2014)

9. Uchida, T., Fukuoka, S., Papanicolaou, A. N. and Takiris, A. G. Non-hydrostatic quasi3D model coupled with dynamic rough wall law for simulating flow over rough bed with submerged boulders, J. Hydraul. Eng. 142 (11), 04016054, 14p. (2016)

10. Chow, V. T. Open Channel Hydraulics, McGraw-Hill, p. 359, (1959)

11. Ohtsu, I. and Yasuda, Y. Transition from supercritical to subcritical flow at an abrupt drop, J. Hydraul. Resear. 29 (3), 309-328 (1991)

12. Nikora, V., McLean, S., Coleman, S., Pokrajac, D., McEwan, I., Campbell, L., Aberle, J., Clunie, D. and Koll, K. : Double-averaging concept for rough-bed open-channel and overland flows: applications, J. Hydraul. Eng. 133, 884-895 (2007)

13. Engelund, F. Flow and bed topography in channel bends, Journal of Hydraulics Division, Proc. of ASCE 100 (11), 1631-1648 (1974) 